\title{
Higher order assortativity in complex networks
}

\author{
Alberto Arcagni, Rosanna Grassi, Silvana Stefani, Anna Torriero ${ }^{\dagger}$
}

\begin{abstract}
Assortativity was first introduced by Newman and has been extensively studied and applied to many real world networked systems since then. Assortativity is a graph metrics and describes the tendency of high degree nodes to be directly connected to high degree nodes and low degree nodes to low degree nodes. It can be interpreted as a first order measure of the connection between nodes, i.e. the first autocorrelation of the degreedegree vector. Even though assortativity has been used so extensively, to the author's knowledge, no attempt has been made to extend it theoretically. This is the scope of our paper. We will introduce higher order assortativity by extending the Newman index based on a suitable choice of the matrix driving the connections. Higher order assortativity will be defined for paths, shortest paths, random walks of a given time length, connecting any couple of nodes. The Newman assortativity is achieved for each of these measures when the matrix is the adjacency matrix, or, in other words, the correlation is of order 1 . Our higher order assortativity indexes can be used for describing a variety of real networks, help discriminating networks having the same Newman index and may reveal new topological network features.
\end{abstract}

Keywords: Assortativity, Degree correlation, Networks, Paths, Random Walks

PACS: $89.75 . \mathrm{Hc}$, 89.75.Fb, 02.50.Ga

\section{Highlights}

- The concept of assortativity is extended to couples of nodes that are not necessarily adjacent but connected through paths, walks, random walks.

${ }^{*}$ Department of Statistics and Quantitative Methods, University of Milano-Bicocca, Milano, Italy

${ }^{\dagger}$ Department of Mathematics, Finance and Econometrics, Catholic University, Milano, Italy 
- A closed formula is found that can be tailored for the higher order assortativity that is of interest for any application at hand.

- The Newman assortativity is shown to be a particular case of all the assortativity measures we propose.

- Simulations show that our approach can help in revealing different topologies in networks with the same Newman assortativity index.

\section{Introduction}

The concept of assortativity was first introduced by Newman ([23] ) and has been studied and applied extensively to any kind of network since. As is known, a network is said to be assortative if nodes of a certain degree tend to be connected to nodes of the same degree, for instance, high degree nodes tend to be connected to high degree nodes and low degree nodes tend to be connected to low degree nodes. A network is said to be disassortative if high degree nodes tend to be connected to low degree nodes. Quoting Newman, "Many networks show "assortative mixing" on their degrees, i.e. a preference for high degree vertices to attach to other high degree vertices. Others show disassortativity mixing high degree vertices attach to low degrees ones". Again "Models that do not take it into account will necessarily fail to reproduce correctly many of the behaviors of real-world networked systems".

We do agree with Newman that assortativity is an important measure and for that we propose an extension that we may call higher order assortativity.

Assortativity is a graph metrics, i.e. related to the topology of the network and is obtained by the Pearson coefficient of the degree-degree correlation vectors. In fact, by definition, the Newman assortativity coefficient focuses on the degree correlation of adjacent nodes, that is, the first autocorrelation of the degree distribution. Based on the adjacency matrix, it can be interpreted as a first order measure of the connection between nodes. The measure is easy to apply and may give useful hints on the network topology (for an extensive review on assortativity see [25]). On the other hand, examples are given in the literature, where networks with apparent different topologies show the same assortativity index, or viceversa, networks with the same apparent topology show different assortativity index (see [9], [1], 2]). This would imply somehow that assortativity is not a "good" measure, but of course it comes from its relative simplicity 
and in our opinion it can be efficiently used for a preliminary inspection to discover the network topology. A way of making assortativity more "efficient" is to extend it theoretically and this is the core of our paper. In fact, our original contribution is meant to extend assortativity to higher order autocorrelations between nodes. We provide a unified approach to assortativity, extending it to higher order connections based on paths, shortest paths and random walks, through suitable definitions of the matrix governing the connections. We provide a closed formula, suitable for all cases we consider. Our formula may be used for measuring assortativity between two not necessarily adjacent nodes but connected through paths, shortest paths or random walks. We show that the Newman assortativity index comes out as a particular case for all the measures we propose. Through simulations we will show that our approach helps in finding synthetic indicators for revealing the network topology. Finally, we provide some hints about choosing the most suitable higher order indicator according to the network at hand and to which flow we are interested to discover. Essentially, extending the concept of assortativity, that is, understanding how high degree nodes are connected to high degree nodes directly or through paths or walks, may help to better understand networks and their topology. As a consequence, if we understand the patterns of interactions, we may leverage this knowledge to improve the flow of knowledge and information (4]). The paper is structured as follows: after the literature review on extensions of assortativity and preliminaries on graph theory (Section 2), we discuss how Newman assortativity is obtained as the first autocorrelation of the degree-degree vector (Subsection 3.1). In the following, higher order assortativity is introduced on random walks (Section 4), paths and geodesics (Section 5). Simulations and conclusions follow.

\subsection{Literature review}

A short literature review on assortativity mixing has been studied extensively since the paper by 23. The original definition of assortativity has been given in [23] for an unweighted and undirected network. Examples of assortativity applied to many real complex networks can also be found in [23]: physics coauthorship, biology coauthorship, film actor collaborations, company directors are examples of assortative networks, while Internet, WWW, protein interaction and neural networks are examples of disassortative networks. Random graphs and Barabasi-Albert networks are examples of non-assortative networks. For a complete and recent review of assortativity we refer to [25]. More generally, in 
F. da Costa et al. ([7]) a survey of measures of complex networks is discussed. Assortativity is sometimes called homophily, when referring specifically to social networks (21]). Among the metrics related to Newman assortativity, we quote the Zagreb Index or $\mathrm{S}(\mathrm{G})$ (1]; 14]). Alderson et al. (1]) discuss the $\mathrm{S}(\mathrm{G}$ ) metric and scale-free graphs. They provide examples of networks having the same degree sequence with an apparent different topology and introduce $S(G)$ as indicators for discriminating network topologies. In [26] many measures are used and accompany assortativity. The authors employ the $d k$-series, a set of characteristics of the network topology, to study the statistical dependencies between different network properties. Through the $d k$-series, they study the average degree, assortativity, clustering and so on. From an empirical point of view, 21] developed and executed an algorithm to evaluate degree correlation between nodes separated by more than one step. Through shortest paths, they studied three online social networks and compared their long range degree correlation behavior to those of three non-social networks by measuring both the average number of neighbors and calculating the Pearson correlation score. Authors conclude that results are not clear cut and require further investigation. Many other measures are used and accompany assortativity, such as in [26].

A discussion on how appropriate the Pearson coefficient is for comparing mixing patterns in networks of a different size can be found in [20]. They show that the Pearson coefficient in scale-free networks decreases with the network size, thus making impossible to compare, for example, two web crawls of different sizes. Alternatively, they suggest a degree-degree dependency measure based on Spearman's rho 19 .

Although assortativity is such an important feature and so popular in the complex networks world, as far as the authors know, no attempts have been made to extend such a measure to a theoretical and unifying view.

\section{Basics about graph theory}

We quickly recall some standard definitions about graph theory. We will assume familiarity with basic theoretical concepts (see [11, [12, 13]). A network is a graph $G=(V, E)$, where $V=\left\{v_{1}, v_{2}, \ldots, v_{n}\right\}$ is the set of vertices and $E \subseteq V \times V$ the set of edges (or links). Let us denote with $|V|=n$ and $|E|=m$ the cardinality of the sets $V$ and $E$, respectively. We consider simple graphs, i.e without loops and multiple edges. An undirected graph is a graph in which if 
$(u, v) \in E$, then $(v, u) \in E$. When two vertices share a link, they are called adjacent. The degree $d_{i}$ of a vertex $v_{i}(i=1, \ldots, n)$ is the number of edges incident with it. We denote by $\mathbf{d}^{T}=\left[\begin{array}{llll}d_{1} & d_{2} & \ldots & d_{n}\end{array}\right]$ the degree sequence of the graph and $\mathbf{D}=\operatorname{diag}\left(d_{i}\right)$. A walk is a sequence of adjacent vertices $v_{1}, v_{2}, \ldots, v_{l}$. A $u-v$ path is a walk connecting $u$ and $v$ in which all vertices are distinct. A shortest path joining vertices $u$ and $v$ is called a $u-v$ geodesic. The distance dist $(u, v)$ between two vertices $u$ and $v$ is the length of the $u-v$ geodesic; the diameter $D$ of $G$ is the maximum of $\operatorname{dist}(u, v), u, v \in V$. A graph is connected if for each pair of vertices $u$ and $v$ there is a path connecting $u$ and $v$. A graph is $k$ - regular if every vertex has the same degree $k$. A graph is bipartite if $V$ can be divided into two separate sets $V_{1}$ and $V_{2}$ such that every node in $V_{1}$ and $V_{2}$ is not connected to each other.

A non-negative $n$-square matrix $\mathbf{A}=\left[a_{i j}\right],(i, j=1,2, \ldots, n)$, representing the adjacency relationships between vertices of $G$, is associated to the graph (the adjacency matrix); the off-diagonal elements $a_{i j}$ of $\mathbf{A}$ are equal to 1 if vertices are adjacent, 0 otherwise. Througout the paper we will assume that all graphs are connected. The matrix $\mathbf{A}$ is said to be primitive if there exists a positive integer $k$ such that $\mathbf{A}^{k}>\mathbf{0}$.

\section{From correlation to assortativity}

Let $\mathbf{x}^{T}=\left[\begin{array}{llll}x_{1} & x_{2} & \ldots & x_{n}\end{array}\right]$ and $\mathbf{y}^{T}=\left[\begin{array}{llll}y_{1} & y_{2} & \ldots & y_{n^{\prime}}\end{array}\right]$ be two real vectors and $\mathbf{E}=\left[e_{i j}\right], 1 \leq i \leq n, 1 \leq j \leq n^{\prime}$ a non-negative matrix of weight 1 between the couples $\left(x_{i}, y_{j}\right)$, such that $\mathbf{1}^{T} \mathbf{E} \mathbf{1}=\sum_{i=1}^{n} \sum_{j=1}^{n^{\prime}} e_{i j}=1$ (where $\mathbf{1}$ is the unit vector). Note that $n$ and $n^{\prime}$ are in general different, but in our case we suppose $n$ equal to $n^{\prime}$.

The sums by columns of the matrix $\mathbf{E}, \mathbf{q}_{\mathbf{x}}=\mathbf{E}^{T} \mathbf{1}$, are the marginal weights of the vector $\mathbf{x}$ and the sums by rows of the matrix $\mathbf{E}, \mathbf{q}_{\mathbf{y}}=\mathbf{E} 1$, are the marginal weights of the vector $\mathbf{y}$.

In order to discuss the Newman assortativity index in the next section, it is useful to recall the definition of the Pearson's linear coefficient (see [13]) and to adapt the notation to our context.

\footnotetext{
${ }^{1}$ In general, the weights can be relative frequencies or probabilities.
} 
Let

$$
\begin{aligned}
\mu_{\mathbf{x}} & =\sum_{i} x_{i} q_{i}^{\mathbf{x}} ; \mu_{\mathbf{y}}=\sum_{j} y_{j} q_{j}^{\mathbf{y}} ; \\
\sigma_{\mathbf{x}} & =\sqrt{\sum_{i}\left(x_{i}-\mu_{\mathbf{x}}\right)^{2} q_{i}^{\mathbf{x}}} ; \sigma_{\mathbf{y}}=\sqrt{\sum_{j}\left(y_{j}-\mu_{\mathbf{y}}\right)^{2} q_{j}^{\mathbf{y}}}
\end{aligned}
$$

be respectively the weighted mean value of $\mathbf{x}$, and $\mathbf{y}$, the weighted standard deviation of $\mathbf{x}$ and $\mathbf{y}$.

The Pearson's linear correlation index is defined by:

$$
r(\mathbf{x}, \mathbf{y})=\frac{\sum_{i} \sum_{j}\left(x_{i}-\mu_{\mathbf{x}}\right)\left(y_{j}-\mu_{\mathbf{y}}\right) e_{i j}}{\sigma_{\mathbf{x}} \sigma_{\mathbf{y}}}
$$

and all the following expressions are equivalent:

$$
\begin{aligned}
r(\mathbf{x}, \mathbf{y}) & =\frac{\sum_{i} \sum_{j} x_{i} y_{j} e_{i j}-\mu_{\mathbf{x}} \mu_{\mathbf{y}}}{\sigma_{\mathbf{x}} \sigma_{\mathbf{y}}}=\frac{1}{\sigma_{\mathbf{x}} \sigma_{\mathbf{y}}}\left[\mathbf{x}^{T} \mathbf{E} \mathbf{y}-\mu_{\mathbf{x}} \mu_{\mathbf{y}}\right]= \\
& =\frac{\sum_{i} \sum_{j} x_{i} y_{j}\left(e_{i j}-q_{i}^{\mathbf{x}} q_{j}^{\mathbf{y}}\right)}{\sigma_{\mathbf{x}} \sigma_{\mathbf{y}}}=\frac{1}{\sigma_{\mathbf{x}} \sigma_{\mathbf{y}}}\left[\mathbf{x}^{T}\left(\mathbf{E}-\mathbf{q}_{\mathbf{x}} \mathbf{q}_{\mathbf{y}}^{T}\right) \mathbf{y}\right]
\end{aligned}
$$

where $\mathbf{q}_{\mathbf{x}}^{T}=\left[\begin{array}{llll}q_{1}^{\mathbf{x}} & q_{2}^{\mathbf{x}} & \ldots & q_{n}^{\mathbf{x}}\end{array}\right]$ and $\mathbf{q}_{\mathbf{y}}^{T}=\left[\begin{array}{llll}q_{1}^{\mathbf{y}} & q_{2}^{\mathbf{y}} & \ldots & q_{n^{\prime}}^{\mathbf{y}}\end{array}\right]$.

By definition, the Pearson's index is the ratio between the covariance of $\mathbf{x}$ and $\mathbf{y}$ and the maximum absolute value that the covariance can assume, i.e. $\sigma_{\mathbf{x}} \sigma_{\mathbf{y}}$ (see formula (1)). Note that $r(\mathbf{x}, \mathbf{y})=0$ occurs in case of absence of correlation. Absence of correlation also occurs in the case in which all the components of one of the two vectors are all equal. However, in this case the ratio takes the form $0 / 0$, then $r(\mathbf{x}, \mathbf{y})$ is undefined.

Observe that, in probability theory (see [12]) $\mathbf{x}$ and $\mathbf{y}$ represent two random variables with $\mathbf{E}$ the matrix of their joint probability distribution. The value $e_{i j}$ is the probability to observe the couple of values $\left(x_{i}, y_{j}\right)$. The vector $\mathbf{q}_{\mathbf{x}}$ is the marginal probability distribution of the variable $\mathbf{x}$. The value $q_{i}^{\mathbf{x}}$ is the probability to observe the value $x_{i}$ without considering the value assumed by the other variable. Analogously, $\mathbf{q}_{\mathbf{y}}$ is the marginal probability distribution of the variable $\mathbf{y}$ and $q_{j}^{\mathbf{y}}$ is the probability to observe the value $y_{j}$ without considering the value assumed by the other variable. Finally, let $\mathbf{D}_{\mathbf{q}_{\mathbf{y}}}=\operatorname{diag}\left(q_{j}^{\mathbf{y}}\right), j=$ $1, . ., n^{\prime}$; observe that, when all the elements of the vector $\mathbf{q}_{\mathbf{y}}$ are positive, this matrix is invertible. $\mathbf{P}_{\mathbf{x} \mid \mathbf{y}}=\mathbf{E} \mathbf{D}_{\mathbf{q}_{\mathbf{y}}}^{-1}=\left[p_{i j}^{\mathbf{x} \mid \mathbf{y}}\right]=\left[e_{i j} / q_{j}^{\mathbf{y}}\right], 1 \leq i \leq n, 1 \leq j \leq n^{\prime}$ 
is a stochastic by column matrix that represents the partial distribution of $\mathbf{x}$ conditioned to the values assumed by the other variable. The value $p_{i j}^{\mathbf{x} \mid \mathbf{y}}$ represents the probability to observe the value $x_{i}$ assuming that the value $y_{j}$ has been observed.

Note that, if the variable $\mathbf{x}$ is independent from the variable $\mathbf{y}$ then $p_{i j}^{\mathbf{x}} \mid \mathbf{y}=q_{i}^{\mathbf{x}}$ for all $1 \leq i \leq n, 1 \leq j \leq n^{\prime}$. Independence is a symmetric relation and it implies $\mathbf{E}=\mathbf{q}_{\mathbf{x}} \mathbf{q}_{\mathbf{y}}^{T}$ having rank equal one. From equation 2 it follows that, if variables $\mathbf{x}$ and $\mathbf{y}$ are independent, $\mathbf{E}-\mathbf{q}_{\mathbf{x}} \mathbf{q}_{\mathbf{y}}^{T}$ is a matrix with entries all equal to zero, then $r(\mathbf{x}, \mathbf{y})=0$.

For later use, let us consider the particular case in which $\mathbf{x}=\mathbf{y}=\mathbf{d}$ and $\mathbf{E}$ a $n \times n$-symmetric matrix; then we get:

$$
\begin{aligned}
\mu_{\mathbf{x}} & =\mu_{\mathbf{y}}:=\mu \\
\sigma_{\mathbf{x}} & =\sigma_{\mathbf{y}}:=\sigma \\
\mathbf{q}_{\mathbf{x}}=\mathbf{q}_{\mathbf{y}} & :=\mathbf{q} .
\end{aligned}
$$

and Pearson's linear correlation index is:

$$
\begin{aligned}
r(\mathbf{d}, \mathbf{d}) & =\frac{\sum_{i} \sum_{j} d_{i} d_{j} e_{i j}-\mu^{2}}{\sigma^{2}}=\frac{1}{\sigma^{2}}\left[\mathbf{d}^{T} \mathbf{E} \mathbf{d}-\mu^{2}\right]= \\
& =\frac{\sum_{i} \sum_{j} d_{i} d_{j}\left(e_{i j}-q_{i} q_{j}\right)}{\sum_{j} d_{j}^{2} q_{j}-\sum_{i} \sum_{j} d_{i} d_{j} q_{i} q_{j}}=\frac{\mathbf{d}^{T}\left(\mathbf{E}-\mathbf{q} \mathbf{q}^{T}\right) \mathbf{d}}{\mathbf{d}^{T}\left(\mathbf{D}_{\mathbf{q}}-\mathbf{q} \mathbf{q}^{T}\right) \mathbf{d}}
\end{aligned}
$$

where $\mathbf{D}_{\mathbf{q}}$ is the diagonal matrix, with diagonal entries equal to the elements of vector $\mathbf{q}$.

\subsection{Newman's assortativity index}

A network is assortative if high degree nodes tend to be connected to high degree nodes, whereas it is disassortative if high degree nodes tend to be connected to low degree nodes. The definition of assortativity was first introduced by Newman ([23]) using the Pearson's coefficient of the degree-degree correlation in an unweighted and undirected network.

Let $G=(V, E)$ be un undirected and unweighted graph, with degree sequence $\mathbf{d}^{T}=\left[\begin{array}{llll}d_{1} & d_{2} & \ldots & d_{n}\end{array}\right]$. Assuming in $3 \mathbf{E}=\frac{1}{2 m} \mathbf{A}$, we get $\mathbf{q}=$ $\frac{1}{2 m} \mathbf{d}, \mathbf{D}_{\mathbf{q}}=\frac{1}{2 m} \mathbf{D}$ and the formula (3) gives the well known measure proposed by Newman, that can be rewritten as: 


$$
\rho=\frac{\mathbf{d}^{T}\left(\frac{\mathbf{A}}{2 m}-\frac{\mathbf{d ~ d}^{T}}{4 m^{2}}\right) \mathbf{d}}{\mathbf{d}^{T}\left(\frac{\mathbf{D}}{2 m}-\frac{\mathbf{d d}^{T}}{4 m^{2}}\right) \mathbf{d}},
$$

or equivalently as (see the Appendix):

$$
\rho=\frac{\frac{1}{2 m} \sum_{i} \sum_{j} d_{i} d_{j} a_{i j}-\left[\frac{1}{4 m} \sum_{i} \sum_{j}\left(d_{i}+d_{j}\right) a_{i j}\right]^{2}}{\frac{1}{4 m} \sum_{i} \sum_{j}\left(d_{i}^{2}+d_{j}^{2}\right) a_{i j}-\left[\frac{1}{4 m} \sum_{i} \sum_{j}\left(d_{i}+d_{j}\right) a_{i j}\right]^{2}} .
$$

Note that (5) is undefined when the graph $G$ is regular, since numerator and denominator are both equal to zero (see [10], pag. 32), or in other words there is no variability within the degree sequence.

The Newman's assortativity coefficient focuses on the degree correlation between only adjacent nodes, so it can be interpreted as a first order measure of the connection between nodes. In this paper, the assortativity definition will be extended also to nodes connected by random walks, paths and shortest paths. All these alternative definitions can be modelled using our unified approach through suitable definitions of matrix $\mathbf{E}$. To this aim, the formula (3) can be used to measure the assortativity between two not necessarily adjacent nodes but connected through random walks, paths and shortest paths.

More in general, $\mathbf{E}$ can be a weight matrix of order $n$, expressing any reciprocal relation between each couple of nodes, giving rise to different indices

of assortativity. In the following Sections, we will introduce higher order assortativity for nodes connected through random walks, paths and shortest paths. For each case, applications taken from the literature will be provided.

\section{Assortativity through random walks}

In this Section a new measure of assortativity based on random walks of length $l$ is introduced. We will show that Newman's assortativity index is only a special case of our measure.

Given the graph $G=(V, E)$, let $E_{w, l} \subseteq V \times V$ be the set of the undirected walks of length $l$. For $l=1, E_{w, 1}=E$ is the set of the edges. Let $\mathbf{E}_{w, l}$ be the matrix of the probabilities that a walk randomly chosen from $E_{w, l}$ connects 
vertices $i$ and $j$. Putting $\mathbf{E}=\mathbf{E}_{w, l}$, the formula (3) can be written as:

$$
\rho_{w, l}=\frac{\mathbf{d}^{T}\left(\mathbf{E}_{w, l}-\mathbf{q q}^{T}\right) \mathbf{d}}{\mathbf{d}^{T}\left(\mathbf{D}_{\mathbf{q}}-\mathbf{q q} \mathbf{q}^{T}\right) \mathbf{d}}
$$

where $\rho_{w, l}$ denotes the linear Pearson coefficient of the degree sequence with weights given by the matrix $\mathbf{E}_{w, l}$. Observe that for $l=1 \mathbf{E}_{w, 1}=\frac{1}{2 m} \mathbf{A}, \mathbf{q}=\frac{1}{2 m} \mathbf{d}$ so that $\rho_{w, 1}$ matches with the Newman measure.

The following result concerns the asymptotic behavior of (6) (the proof is reported in the Appendix):

Theorem 1 Let $G=(V, E)$ be a graph with adjacency matrix $\mathbf{A}$ and degree sequence $\mathbf{d}$. Let $\mathbf{P}$ be the transition matrix of a Markov chain on $G=(V, E)$. If $\mathbf{P}$ is primitive, the assortativity of order $l, \rho_{w, l}$ vanishes as $l \rightarrow \infty$.

Observe that, for bipartite connected graphs, $\mathbf{P}$ is not primitive. In this case, $\mathbf{P}^{l}$ does not converge to the stationary distribution (see [24], [17]). Moreover, similarly to the Newman assortativity (5), if $G=(V, E)$ is a regular graph, the assortativity of order $l\left(\rho_{w, l}\right)$ is undefined.

A possible application of higher order assortativity through random walks can be found in an input-ouput network (see [5]), where the movement of goods between the sectors of an economy is modeled as a random walk. Goods, like random walkers, start out at a given position and repeatedly choose an edge incident to their current position. The choices are made according to a probability distribution determined by the edge weights. The goods proceed for an arbitrarily long time or until a prescribed goal is reached. From formula (6) we may be able to track down the movements of those goods.

\section{Assortativity through paths}

In this Section we define a new measure of assortativity based on paths of length $l$. The Newman assortativity measure will be extended by taking into account not only the direct connection between two nodes (i.e. the adjacency) but also the higher order neighborhood structure of the network through paths.

Being the graph $G$ connected, two nodes $i$ and $j$ are always linked by a path of some length $l$. However, an assortativity measure should also capture more complex structural features, such as the degree of the nodes belonging to the 
$(i, j)$-path. This can be obtained by (3) by a suitable choice of the matrix $\mathbf{E}$ as explained in this Section.

\subsection{Assortativity through degree-based paths}

Given the graph $G=(V, E)$, let $E_{p, l} \subseteq V \times V$ be the set of undirected paths of length $l$ between any couple of nodes. Let $\mathbf{E}_{p, l}$ be the weighted matrix associated to $E_{p, l}$, whose entries are $e_{i j}=\frac{1}{e} \sum_{i_{1} i_{2} \ldots i_{l-1}}\left(d_{i_{1}} d_{i_{2}} \ldots . d_{i_{l-1}}\right), i, j=1, \ldots, n$, where $i_{1} i_{2} \ldots i_{l-1}$ are the nodes belonging to all $l$ - paths between $i$ and $j$ and $e$ is the sum of $d_{i_{1}} d_{i_{2}} \ldots . . d_{i_{l-1}}$ over all the $l$ - paths in $E_{p, l}$.

Assuming $\mathbf{E}=\mathbf{E}_{p, l}$, formula (3) becomes:

$$
\rho_{p, l}=\frac{\mathbf{d}^{T}\left(\mathbf{E}_{p, l}-\mathbf{q q}^{T}\right) \mathbf{d}}{\mathbf{d}^{T}\left(\mathbf{D}_{\mathbf{q}}-\mathbf{q q} \mathbf{q}^{T}\right) \mathbf{d}} .
$$

A particular case occurs when $l=1$, i.e. the path lengths are all equal to 1. Then $E_{p, 1}$ becomes the set of edges $E, \mathbf{E}_{p, 1}=\frac{1}{2 m} \mathbf{A}$, since $e$ reduces to the sum of the degrees $(2 m)$. Vector $\mathbf{q}$ becomes $\frac{1}{2 m} \mathbf{d}$, so that $\rho_{p, 1}$ corresponds to the Newman measure. Notice that, unlike assortativity through random walks, (7) exists only for $l$ lower or equal to the length of the longest path.

Let $\mathbf{E}_{u p, l}$ be the matrix associated to the set of undirected paths of length $l$ obtained by $\mathbf{E}_{p, l}$ putting elements $d_{i_{k}}=1$, where $i_{1} i_{2} \ldots . i_{l-1}$ are the nodes belonging to all $l$ - paths between $i$ and $j$. The entries $e_{i j}$ of this matrix simply become the number of the existing $l$ - path between nodes $i$ and $j$, divided by $e$ (simply the number of all $l$ - paths in $E_{p, l}$ ).

Formula (7) can be rewritten as:

$$
\rho_{u p, l}=\frac{\mathbf{d}^{T}\left(\mathbf{E}_{u p, l}-\mathbf{q q}^{T}\right) \mathbf{d}}{\mathbf{d}^{T}\left(\mathbf{D}_{\mathbf{q}}-\mathbf{q q}^{T}\right) \mathbf{d}} .
$$

In this case, assortativity extends the degree correlations beyond adjacency through paths of a given length, but keeping into account only the degree of the source and the target nodes.

This assortativity is related to a topological index, the higher-order connectivity index, extensively used in Chemistry (see [16]). The higher order connectivity index was proposed in the literature as a generalization of the Randic index (see [29]). 
Following the definition found in [18, for an integer $l \geq 1$, the $l$ - connectivity index is defined as:

$$
\chi_{\alpha}^{l}(G)=\sum_{(i, j)}\left(d_{i} d_{i_{1}} d_{i_{2}} \ldots d_{i_{l-1}} d_{j}\right)^{\alpha}
$$

$\alpha>0$, where the sum runs over all $(i-j)$ paths of length $l$ of $G$.

The higher-order connectivity index had various chemical applications, but so far not many mathematical results have been obtained on $\chi_{\alpha}^{l}(G)$; some results can be found in [18, [28, and [31.

Observe that in (7) and (8) the first term on the numerator, i.e. $\mathbf{d}^{T} \mathbf{E}_{p, l} \mathbf{d}$ and $\mathbf{d}^{T} \mathbf{E}_{\text {up }, \mathrm{l}} \mathbf{d}$, differs from the $l$-connectivity index when the exponent respectively is $\alpha=1$ and $\alpha=0$ by the multiplicative factor $\frac{1}{e}$.

\subsection{Assortativity through shortest paths}

From formula (3), a measure of assortativity, based on shortest paths of length $l \leq D$, can also be defined. Let $E_{s p, l} \subseteq V \times V$ the set of geodesics of length $l$ and $\mathbf{E}_{s p, l}$ be its associated matrix, whose entries $e_{i j}$ are defined as in (8) where $i_{1} i_{2} \ldots i_{l-1}$ are the nodes belonging to all $l$ - shortest paths between $i$ and $j$, divided by the cardinality of $E_{s p, l}$. Formula (3) becomes in this case:

$$
\rho_{s p, l}=\frac{\mathbf{d}^{T}\left(\mathbf{E}_{s p, l}-\mathbf{q} \mathbf{q}^{T}\right) \mathbf{d}}{\mathbf{d}^{T}\left(\mathbf{D}_{\mathbf{q}}-\mathbf{q} \mathbf{q}^{T}\right) \mathbf{d}}
$$

observe that this index has been proposed by Mayo et al. in [21].

Another assortativity measure, based on shortest paths, can be defined in the following way.

Given the connected graph $G=(V, E)$, let $\mathbf{H}_{\alpha}=\left[h_{i j}\right]$, be the matrix having the diagonal entries equal to zero, whereas $h_{i j}=\operatorname{dist}(i, j)^{-\alpha}$ for $i \neq j, \alpha>0$ real parameter. Assuming in $3 \mathbf{E}=\frac{1}{h} \mathbf{H}_{\alpha}$, where $h=\sum_{i} \sum_{j} h_{i j}$, we obtain:

$$
\rho_{c, \alpha}=\frac{\mathbf{d}^{T}\left(\frac{1}{h} \mathbf{H}_{\alpha}-\mathbf{q} \mathbf{q}^{T}\right) \mathbf{d}}{\mathbf{d}^{T}\left(\mathbf{D}_{\mathbf{q}}-\mathbf{q} \mathbf{q}^{T}\right) \mathbf{d}}=\frac{\frac{1}{h} \mathbf{d}^{T} \mathbf{H}_{\alpha} \mathbf{d}-\mathbf{d}^{T} \mathbf{q} \mathbf{q}^{T} \mathbf{d}}{\mathbf{d}^{T}\left(\mathbf{D}_{\mathbf{q}}-\mathbf{q} \mathbf{q}^{T}\right) \mathbf{d}} .
$$

Differently from the previous indices, $\rho_{c, \alpha}$ measures assortativity also taking into account, in addition to the degree sequence, the length of the shortest path between nodes $i$ and $j$, i.e. dist $(i, j)$. This measure generalizes Newman assortativity, including all couples of nodes (adjacent or not) in the formula but 
with decreasing weights as the distance between them increases.

We can prove the following Theorem (see the Appendix 2):

Theorem 2 Let $G=(V, E)$ be a simple connected graph. The coefficient $\rho_{c, \alpha}$ tends to $\rho$ as $\alpha$ tends to infinity.

Observe that formula (9) is related to another global network indicator, known in the literature as clumpiness (see [8]). Given a connected graph $G=$ $(V, E)$, the clumpiness coefficient is defined by:

$$
\Lambda(G, \mathbf{d}, \alpha)=\sum_{i>j}^{n(n-1) / 2} \frac{d_{i} d_{j}}{(\operatorname{dist}(i, j))^{\alpha}}=\frac{1}{2} \mathbf{d}^{T} \mathbf{H}_{\alpha} \mathbf{d} .
$$

It is easy to observe that $\rho_{c, \alpha}$ can also be written as:

$$
\rho_{c, \alpha}=\frac{\frac{2}{h} \Lambda(G, \mathbf{d}, \alpha)-\mathbf{d}^{T} \mathbf{q} \mathbf{q}^{T} \mathbf{d}}{\mathbf{d}^{T} \mathbf{D}_{\mathbf{q}} \mathbf{d}-\mathbf{d}^{T} \mathbf{q} \mathbf{q}^{T} \mathbf{d}} .
$$

Note that, as the authors point out in ([8]), clumpiness and the Newman's index measure different features of the network. Indeed, the clumpiness index $\Lambda(G, \mathbf{d}, \alpha)$ increases with the increase of the node degrees but, on the contrary, it decreases with the increase in the distance between them, and various examples of clumped assortative and clumped disassortative networks are provided in (8).

A classic example of a flow moving through geodesics in a network is given by logistics. A driver delivering a package normally knows and selects the shortest route possible, so that the package's trajectory follows geodesic paths through the road network [6]. The higher order assortativity based on shortest paths may help to track down the flow of packages.

\section{Simulations}

In this section some simulations are performed in order to analyse and compare the different assortativity measures previously defined.

We simulate 100 graphs of same order $n=30$ with the same degree sequence:

$$
\mathbf{d}=\left[7^{(3)}, 6^{(2)}, 5^{(8)}, 4^{(3)}, 3^{(8)}, 2^{(5)}, 1^{(1)}\right] .
$$

Graphs are connected, non-isomorphic and without loops. They have same size 


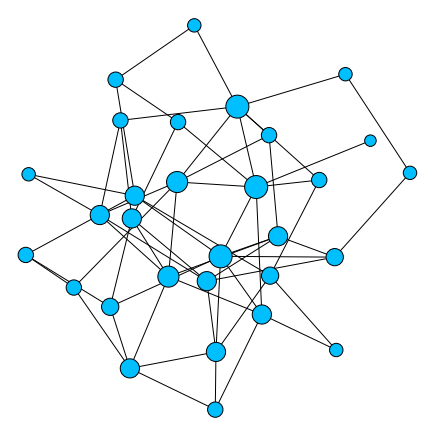

(a) Graph $G_{1}$

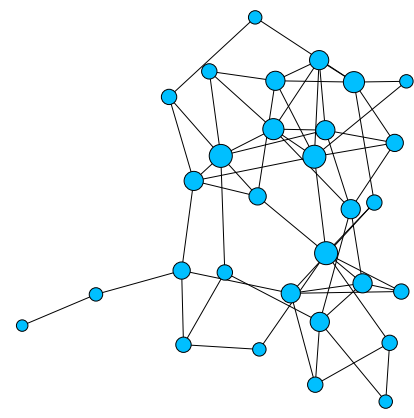

(b) Graph $G_{2}$

Figure 1: Two simulated graphs with the same Newman's coefficient.

$m=60$, average degree $\mu=4$ and density $\delta=\frac{2 m}{n(n-1)}=0.1379$, but they differ by topology.

Let us consider the simulated graphs, $G_{1}, G_{2}$ and $G_{3}, G_{4}$, respectively in Figures 1 and 3 (higher degree nodes are thicker).

Graphs $G_{1}$ and $G_{2}$ have the same Newman's index, $\rho\left(G_{1}\right)=\rho\left(G_{2}\right)=$ -0.0584 and they are equally disassortative but, by inspection of Figure 1 , they are topologically different. Indeed, we will see that other structural parameters, based on shortest paths, give different values.

To this end we check some classical network indicators: the diameter $D$, the average (shortest) path length:

$$
L=\frac{1}{n(n-1)} \sum_{i \neq j} \operatorname{dist}(i, j)
$$

and the clustering coefficient, also named transitivity (see [22]):

$$
C=\frac{3|T(3)|}{P_{2}}
$$

where $T(3)$ is the number of triangles and $P_{2}$ is the number of 2-paths.

Observe that $D\left(G_{1}\right)=4$, whereas $D\left(G_{2}\right)=6 ; L\left(G_{1}\right)=2.4483$ whereas $L\left(G_{2}\right)=2.6230 . C\left(G_{1}\right)=0.1091$ whereas $C\left(G_{2}\right)=0.1773$. 
Table 1: Higher order assortativity measures of graphs $G_{1}$ and $G_{2}$.

\begin{tabular}{r|rrr|rrr}
$l$ & $\rho_{w, l}\left(G_{1}\right)$ & $\rho_{p, l}\left(G_{1}\right)$ & $\rho_{u p, l}\left(G_{1}\right)$ & $\rho_{w, l}\left(G_{2}\right)$ & $\rho_{p, l}\left(G_{2}\right)$ & $\rho_{u p, l}\left(G_{2}\right)$ \\
\hline 1 & -0.0584 & -0.0584 & -0.0584 & -0.0584 & -0.0584 & -0.0584 \\
2 & 0.1826 & -0.0787 & -0.0864 & 0.3501 & 0.0951 & 0.1024 \\
3 & -0.0322 & -0.0593 & -0.0563 & 0.0183 & -0.0181 & -0.0054 \\
4 & 0.0719 & 0.0030 & 0.0010 & 0.1796 & -0.0661 & -0.0495 \\
5 & -0.0245 & -0.0306 & -0.0316 & 0.0319 & -0.0348 & -0.0309 \\
6 & 0.0347 & -0.0376 & -0.0336 & 0.1066 & -0.0494 & -0.0457 \\
7 & -0.0169 & -0.0486 & -0.0403 & 0.0301 & -0.0549 & -0.0538 \\
8 & 0.0185 & -0.0452 & -0.0390 & 0.0680 & -0.0532 & -0.0573 \\
9 & -0.0110 & 0.0379 & -0.0334 & 0.0247 & -0.0497 & -0.0543 \\
10 & 0.0105 & -0.0397 & -0.0361 & 0.0451 & -0.0489 & -0.0543
\end{tabular}

Table 1 reports the assortativity measures proposed in this papen referring to $G_{1}$ and $G_{2}$, allowing us to analyze all the measures simultaneoulsy.

Furthermore, the diagrams in Figures (2) from (a) to (f) depict the plot of proposed assortativity measures, also shown in Table 1, for different values of lengths $l$, allowing us to focus on each measure separately. First of all, as we previously proved, all measures for $l=1$ correspond to the Newman's index. Moreover, the assortativity through random walk vanishes as $l$ approaches to infinity.

Looking at the values in Table 1, referring to the Newman's index, graphs are equally, slightly disassortative so, for both, high degree nodes tend to be adjacent to low degree nodes. However, looking at the assortativity beyond the nearest neighbors, the two graphs are different and our measures are able to better capture the topological features related to the assortativity.

Taking as an example $l=2, G_{2}$ is certainly assortative, referring to all the measures, as $\rho_{w, 2}\left(G_{2}\right)=0.3501, \rho_{p, 2}\left(G_{2}\right)=0.0951, \rho_{u p, 2}\left(G_{2}\right)=0.1024$, and this is due to the prevalence of existing connections between similar degree nodes through 2 steps. On the contrary, this effect is not present for $G_{1}$, that shows assortativity through random walks of length 2 but not through 2-paths, as $\rho_{w, 2}\left(G_{1}\right)=0.1826, \rho_{p, 2}\left(G_{1}\right)=-0.0787, \rho_{u p, 2}\left(G_{1}\right)=-0.0864$. This result is consistent with the transitivity values, being $C\left(G_{2}\right)$ higher than $C\left(G_{1}\right)$.

For $l=3, G_{1}$ certainly becomes disassortative, referring to all the measures, as $\rho_{w, 3}\left(G_{1}\right), \rho_{p, 3}\left(G_{1}\right), \rho_{u p, 3}\left(G_{1}\right)$ are negative, prevailing the connections between

\footnotetext{
${ }^{2}$ The number of undirected paths of length $l=10$ is of order $10^{5}$ of magnitude and our R-code 27 uses a recursive algorithm in order to extract the undirected paths, making the procedure computationally intensive. For this reason, the measures depending on lengths $l$ have been evaluated until $l=10$.
} 
Table 2: Higher order assortativity measures of graphs $G_{3}$ and $G_{4}$.

\begin{tabular}{r|rrr|rrr}
$l$ & $\rho_{w, l}\left(G_{3}\right)$ & $\rho_{p, l}\left(G_{3}\right)$ & $\rho_{u p, l}\left(G_{3}\right)$ & $\rho_{w, l}\left(G_{4}\right)$ & $\rho_{p, l}\left(G_{4}\right)$ & $\rho_{u p, l}\left(G_{4}\right)$ \\
\hline 1 & 0.0966 & 0.0966 & 0.0966 & 0.0966 & 0.0966 & 0.0966 \\
2 & 0.2389 & 0.0198 & 0.0165 & 0.2307 & -0.1229 & -0.1037 \\
3 & -0.0071 & -0.1209 & -0.1171 & 0.1019 & -0.0601 & -0.0449 \\
4 & 0.0808 & -0.1192 & -0.1107 & 0.1258 & -0.0339 & -0.0374 \\
5 & -0.0109 & -0.0656 & -0.0531 & 0.0816 & -0.0291 & -0.0298 \\
6 & 0.0334 & -0.0565 & -0.0511 & 0.0850 & -0.0361 & -0.0374 \\
7 & -0.0059 & -0.0445 & -0.0408 & 0.0623 & -0.0375 & -0.0403 \\
8 & 0.0156 & -0.0438 & -0.0430 & 0.0615 & -0.0352 & -0.0378 \\
9 & -0.0025 & -0.0465 & -0.0469 & 0.0472 & -0.0383 & -0.0394 \\
10 & 0.0079 & -0.0491 & -0.0459 & 0.0457 & -0.0422 & -0.0420
\end{tabular}

high degree nodes with low degree nodes through 3 steps. $G_{2}$ shows assortativity through random walks of length 3 and disassortativity through 3-paths.

Looking at the diagrams in Figures (2 a-b), also for $l=2$, both graphs are assortative, in particular $G_{2}$ is more assortative than $G_{1}$, whereas for $l=3, G_{1}$ becomes disassortative and $G_{2}$ still assortative. In general, we can deduce that, for $G_{2}$, similar degree nodes tend to be connected to each other by walks of any length $l>1$, indeed $\rho_{w, l}\left(G_{2}\right)$ assumes positive signs. On the contrary, $\rho_{w, l}\left(G_{1}\right)$ presents an alternating sequence of signs. It is worth noting that, degree-based paths (Figures 2 c-f) do not significantly modify correlations and, for a given graph and a given length, $\rho_{p, l}$ and $\rho_{u p, l}$ are quite similar.

A similar analysis can be done for graphs $G_{3}$ and $G_{4}$ (see Figure 3 ).

They share the same Newman's index $\rho\left(G_{3}\right)=\rho\left(G_{4}\right)=0.0966$ so in this case they are both assortative. Classical network indicators are $D\left(G_{3}\right)=5$, whereas $D\left(G_{4}\right)=7 ; L\left(G_{3}\right)=2.5931$ whereas $L\left(G_{4}\right)=2.7448 . C\left(G_{3}\right)=0.1091$ whereas $C\left(G_{4}\right)=0.2045$.

Observing the values referred to graph $G_{3}$ in Table (2), assortativity is also confirmed for $l=2$, as $\rho_{w, 2}\left(G_{3}\right), \rho_{p, 2}\left(G_{3}\right), \rho_{u p, 2}\left(G_{3}\right)$ are positive, whereas $G_{3}$ becomes disassortative for $l=3$.

On the contrary, assortativity is not confirmed for graph $G_{4}$ showing assortativity through random walks of length $l>1$ but not through $l$-paths. Notice that, in this case, results are not consistent with the transitivity values, being now $C\left(G_{3}\right)$ lower than $C\left(G_{4}\right)$.

Looking at Figure (4 a-b), the measures of assortativity through random walks of length 2 are similar, being $\rho_{w, 2}\left(G_{3}\right)=0.2389$ and $\rho_{w, 2}\left(G_{4}\right)=0.2307$. Therefore, in order to identify a graph through these measures it is convenient 


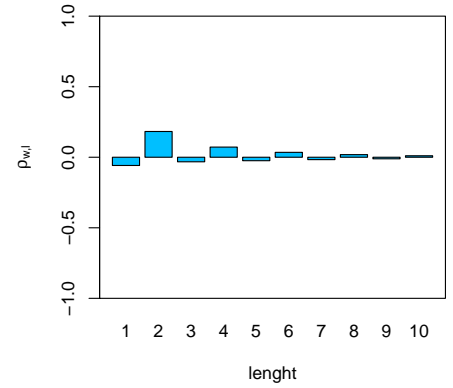

(a) Assortativity through random walks of $G_{1}$

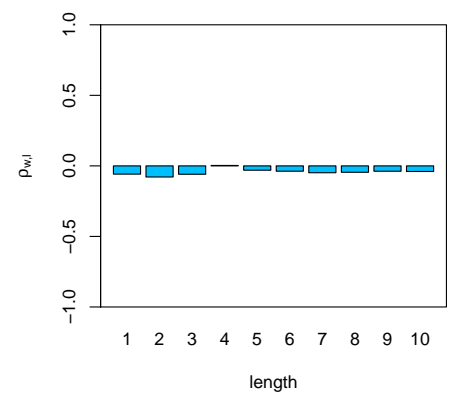

(c) Assortativity through degreebased paths of $G_{1}$

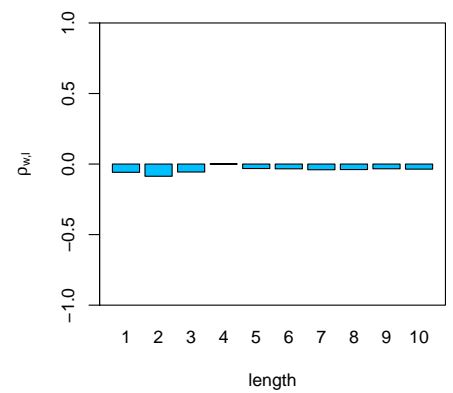

(e) Assortativity through paths of $G_{1}$

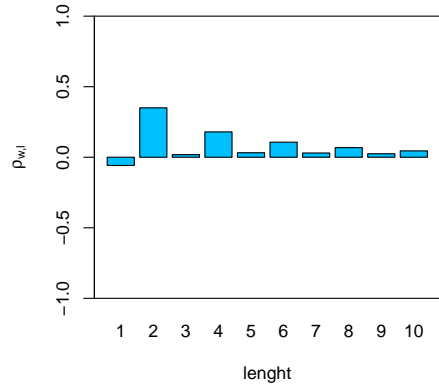

(b) Assortativity through random walks of $G_{2}$

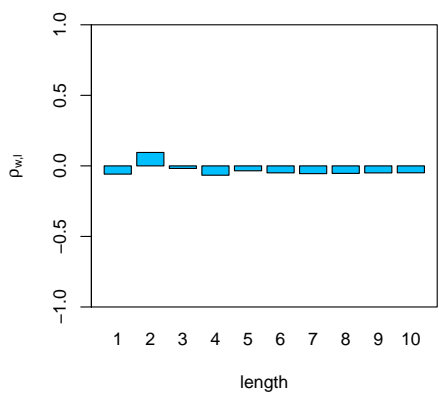

(d) Assortativity through degreebased paths of $G_{2}$

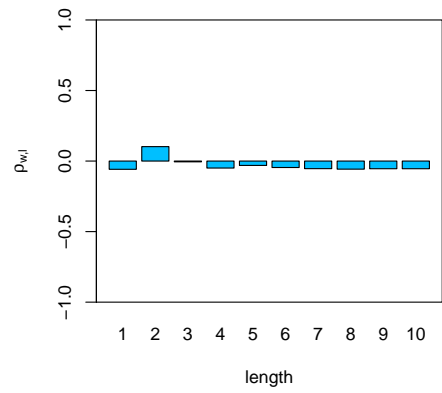

(f) Assortativity through paths of $G_{2}$

Figure 2: Plot of higher order of assortativity measures of graphs $G_{1}$ and $G_{2}$ depending on length $l$. 


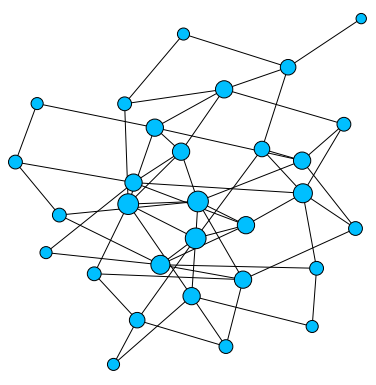

(a) Graph $G_{3}$

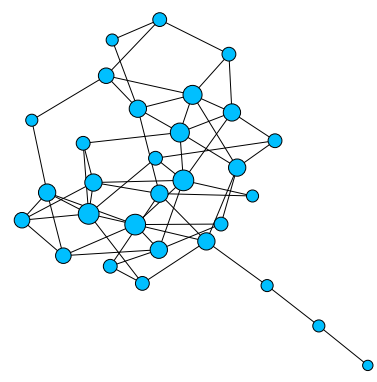

(b) Graph $G_{4}$

Figure 3: Two simulated graphs with the same Newman's coefficient.

to consider them for all lengths, taking into account the class of the measures as a whole. The behavior becomes completely different taking into account longer random walks $(l>2)$.

Unlike the other indices we proposed, $\rho_{c, \alpha}(\cdot)$ (assortativity through shortest paths) is not a function of path lengths therefore plots are not provided. It is a measure that summarizes the graph assortativity for different orders of lengths. In particular, distance between vertices is used as weight to reduce the influence of distant vertices in the evaluation of assortativity through all the couples of connected vertices. In this example we evaluated the measure for $\alpha=1$. A larger $\alpha$ reduces the relevance of vertices that are not adjacent, so that the Newman index is the limit of $\rho_{c, \alpha}(\cdot)$ as $\alpha$ approaches infinity. Therefore a comparison between $\rho_{c, 1}(\cdot)$ and $\rho(\cdot)$ is useful to understand the influence on the assortativity of vertices that are connected but not adjacent.

The Newman index is equal and negative for both graphs $G_{1}$ and $G_{2}\left(\rho\left(G_{1}\right)=\right.$ $\left.\rho\left(G_{2}\right)=-0.0584\right)$ and the measure through shortest paths is $\rho_{c, 1}\left(G_{1}\right)=-0.0327$ and $\rho_{c, 1}\left(G_{2}\right)=-0.0089$. Therefore we can assert that both graphs are disassortative (vertices with large degrees tend to be connected to vertices with low degrees) and that farther vertices reduce the intensity of such relations.

The case of graphs $G_{3}$ and $G_{4}$ is more particular. Newman index asserts that both graphs are equally assortative, $\rho\left(G_{3}\right)=\rho\left(G_{4}\right)=0.0966$, but if we consider the synthetic measure through shortest paths we observe that this 


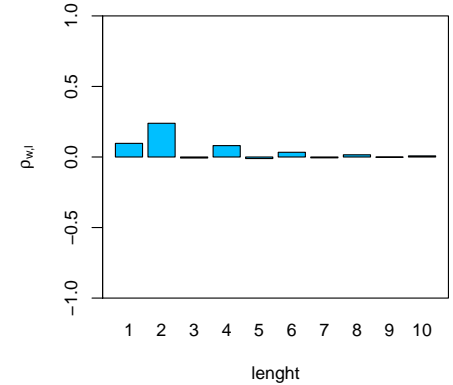

(a) Assortativity through random walks of $G_{3}$

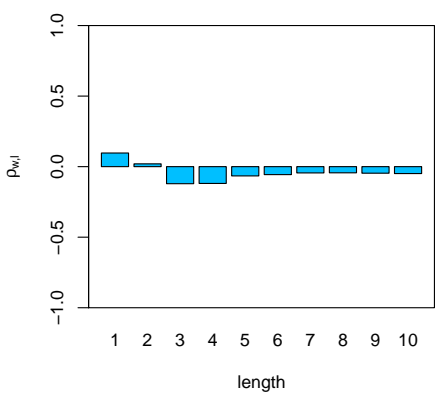

(c) Assortativity through degreebased paths of $G_{3}$

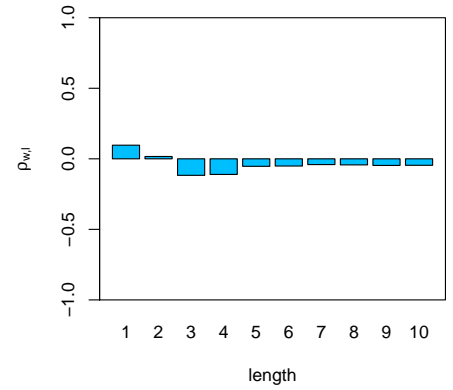

(e) Assortativity through paths of $G_{3}$

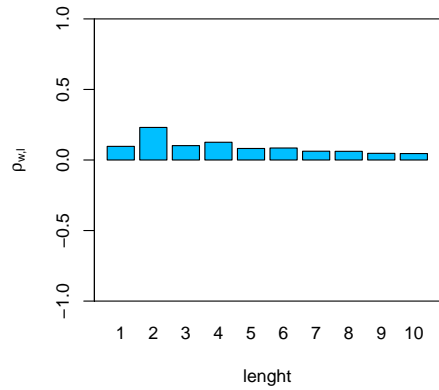

(b) Assortativity through random walks of $G_{4}$

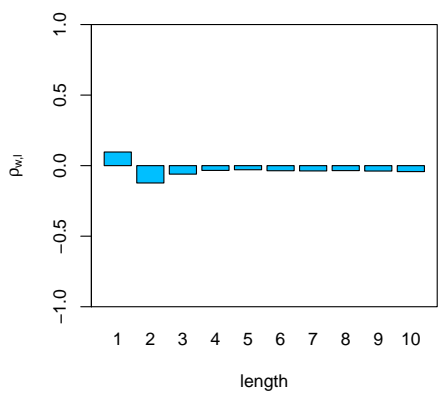

(d) Assortativity through degreebased paths of $G_{4}$

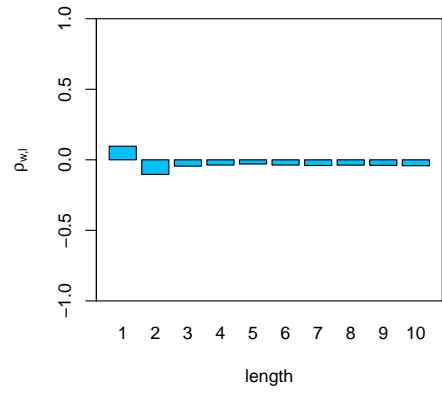

(f) Assortativity through paths of $G_{4}$

Figure 4: Assortativity measures of graphs $G_{3}$ and $G_{4}$ 
relation vanishes and they become almost disassortative, $\rho_{c, 1}\left(G_{3}\right)=-0.0074$ $\rho_{c, 1}\left(G_{4}\right)=-0.0035$. This means that, even if vertices tend to be adjacent with other similar vertices, when we also consider vertices connected by a larger distance there is no linear relation between vertices degree because the measures are close to zero.

\section{Conclusions}

Using a unified approach, in this paper we have introduced high order assortativity based on paths, shortest paths and random walks. The analysis has been performed for undirected and unweighted networks. Through simulations, we have shown that higher order assortativity can help to better reveal the network topology. The analysis can be possibly extended to weighted and directed networks.

\section{Appendix 1}

\section{Newman's assortativity index}

Here we report all mathematical details needed to obtain the Newman's formula (5) from expression (4):

$$
\begin{aligned}
\rho & =\frac{\mathbf{d}^{T}\left(\frac{\mathbf{A}}{2 M}-\frac{\mathbf{d} \mathbf{d}^{T}}{4 M^{2}}\right) \mathbf{d}}{\mathbf{d}^{T}\left(\frac{\mathbf{D}}{2 M}-\frac{\mathbf{d} \mathbf{d}^{T}}{4 M^{2}}\right) \mathbf{d}}=\frac{\frac{1}{2 M} \mathbf{d}^{T} \mathbf{A} \mathbf{d}-\frac{1}{4 M^{2}} \mathbf{d}^{T}\left(\mathbf{d} \mathbf{d}^{T}\right) \mathbf{d}}{\mathbf{d}^{T} \frac{\mathbf{D}}{2 M} \mathbf{d}-\frac{1}{4 M^{2}}\left(\mathbf{d}^{T} \mathbf{d} \mathbf{d}^{T} \mathbf{d}\right)}= \\
& =\frac{\frac{1}{2 M} \mathbf{d}^{T} \mathbf{A} \mathbf{d}-\frac{1}{4 M^{2}}\left(\mathbf{d}^{T} \mathbf{d}\right)^{2}}{\frac{1}{2 M} \mathbf{d}^{T} \mathbf{D} \mathbf{d}-\frac{1}{4 M^{2}}\left(\mathbf{d}^{T} \mathbf{d}\right)^{2}} .
\end{aligned}
$$

Observe now that:

$$
\mathbf{d}^{T} \mathbf{A d}=\sum_{i} d_{i}\left(\sum_{j} a_{i j} d_{j}\right)=\sum_{i} \sum_{j} d_{i} d_{j} a_{i j}=2 \sum_{i \sim j} d_{i} d_{j}
$$

since in the left-hand side, the sum is over all possible couples $(i, j)$, whereas on the right-hand side, the sum is over all adjacent couples $(i, j)$ and nodes $i$ and $j$ are counted twice. 
Now it is easy to check the following chain of equalities:

$$
\mathbf{d}^{T} \mathbf{d}=\sum_{i} d_{i}^{2}=\sum_{i \sim j}\left(d_{i}+d_{j}\right)=\sum_{i} \sum_{j} \frac{1}{2}\left(d_{i}+d_{j}\right) a_{i j}
$$

Indeed, the summation of $\left(d_{i}+d_{j}\right)$ is over all couples of adjacent nodes, so that every term $d_{i}$ appears as much times as its degree, i.e. $d_{i}$ times.

Similar argument leads to the following chain of equality:

$$
\mathbf{d}^{T} \mathbf{D} \mathbf{d}=\sum_{i} d_{i}^{3}=\sum_{i \sim j}\left(d_{i}^{2}+d_{j}^{2}\right)=\sum_{i} \sum_{j} \frac{1}{2}\left(d_{i}^{2}+d_{j}^{2}\right) a_{i j} .
$$

Then the numerator becomes:

$$
\begin{aligned}
\frac{1}{2 M} \mathbf{d}^{T} \mathbf{A d}-\frac{1}{4 M^{2}}\left(\mathbf{d}^{T} \mathbf{d}\right)^{2} & =\frac{2 \sum_{i \sim j} d_{i} d_{j}}{2 M}-\frac{\left(\sum_{i \sim j}\left(d_{i}+d_{j}\right)\right)^{2}}{4 M^{2}}= \\
& =\frac{1}{M} \sum_{i \sim j} d_{i} d_{j}-\left[\frac{1}{2 M} \sum_{i \sim j}\left(d_{i}+d_{j}\right)\right]^{2}= \\
& =\frac{1}{2 M} \sum_{i} \sum_{j} d_{i} d_{j} a_{i j}-\left[\frac{1}{4 M} \sum_{i} \sum_{j}\left(d_{i}+d_{j}\right) a_{i j}\right]^{2}
\end{aligned}
$$

The denominator is:

$$
\begin{aligned}
\frac{1}{2 M} \mathbf{d}^{T} \mathbf{D} \mathbf{d}-\frac{1}{4 M^{2}}\left(\mathbf{d}^{T} \mathbf{d}\right)^{2} & =\frac{1}{2 M} \sum_{i \sim j}\left(d_{i}^{2}+d_{j}^{2}\right)-\frac{\left(\sum_{i \sim j}\left(d_{i}+d_{j}\right)\right)^{2}}{4 M^{2}}= \\
& =\frac{1}{2 M} \sum_{i \sim j}\left(d_{i}^{2}+d_{j}^{2}\right)-\left[\frac{1}{2 M} \sum_{i \sim j}\left(d_{i}+d_{j}\right)\right]^{2}= \\
& =\frac{1}{4 M} \sum_{i} \sum_{j}\left(d_{i}^{2}+d_{j}^{2}\right) a_{i j}-\left[\frac{1}{4 M} \sum_{i} \sum_{j}\left(d_{i}+d_{j}\right) a_{i j}\right]^{2}
\end{aligned}
$$

yielding to the final formula:

$$
\rho=\frac{\frac{1}{2 M} \sum_{i} \sum_{j} d_{i} d_{j} a_{i j}-\left[\frac{1}{4 M} \sum_{i} \sum_{j}\left(d_{i}+d_{j}\right) a_{i j}\right]^{2}}{\frac{1}{4 M} \sum_{i} \sum_{j}\left(d_{i}^{2}+d_{j}^{2}\right) a_{i j}-\left[\frac{1}{4 M} \sum_{i} \sum_{j}\left(d_{i}+d_{j}\right) a_{i j}\right]^{2}} .
$$


and its equivalent forms:

$$
\begin{aligned}
\rho & =\frac{\frac{1}{M} \sum_{i \sim j} d_{i} d_{j}-\left[\frac{1}{2 M} \sum_{i \sim j}\left(d_{i}+d_{j}\right)\right]^{2}}{\frac{1}{2 M} \sum_{i \sim j}\left(d_{i}^{2}+d_{j}^{2}\right)-\left[\frac{1}{2 M} \sum_{i \sim j}\left(d_{i}+d_{j}\right)\right]^{2}}= \\
& =\frac{\sum_{i \sim j} d_{i} d_{j}-\left[\sum_{i} \frac{1}{2} d_{i}^{2}\right]^{2} / M}{\sum_{i} \frac{1}{2} d_{i}^{3}-\left[\sum_{i} \frac{1}{2} d_{i}^{2}\right]^{2} / M} .
\end{aligned}
$$

\section{Appendix 2}

\section{Proof of Theorem 1}

Let $G=(V, E)$ be a graph with adjacency matrix $\mathbf{A}$ and degree sequence $\mathbf{d}$. Let $\mathbf{P}$ be the transition matrix of a Markov chain on $G=(V, E)$. If $\mathbf{P}$ is primitive, the assortativity of order $l, \rho_{w, l}$ vanishes as $l \rightarrow \infty$.

Proof. Let us now consider a Markov chain on $G=(V, E)$ with adjacency matrix $\mathbf{A}$ and degree sequence $\mathbf{d}$ (see [15]) and let $\mathbf{P}$ be the transition matrix. First of all, $\mathbf{P}^{l}$ provides the partial probability distributions of being at the $j$-th state of the Markov chain after $l$ steps starting from the $i$-th state, then $\mathbf{E}_{w, l}=\mathbf{D}_{\mathbf{q}} \mathbf{P}^{l}$, i.e. the probabilities that a walk randomly chosen from $E_{w, l}$ connects vertices $i$ and $j$ can be obtained by multiplying each partial distribution by the probability to be in the $i$-th state. Observe that also for $l>1$ it holds $\mathbf{q}=\frac{1}{2 m} \mathbf{d}$, for a well known property of Markov chain on undirected graphs.

As a consequence, the measure of assortativity of order $l$ is:

$$
\rho_{w, l}=\frac{\mathbf{d}^{T}\left(\mathbf{D}_{\mathbf{q}} \mathbf{P}^{l}-\mathbf{q q}^{T}\right) \mathbf{d}}{\mathbf{d}^{T}\left(\mathbf{D}_{\mathbf{q}}-\mathbf{q} \mathbf{q}^{T}\right) \mathbf{d}}
$$

Recalling that $\mathbf{P}=\mathbf{D}^{-1} \mathbf{A}$ and $\mathbf{D}_{\mathbf{q}}=\frac{1}{2 m} \mathbf{D}, 10$ can be rewritten as:

$$
\rho_{w, l}=\frac{\mathbf{d}^{T}\left(\frac{\mathbf{D}\left(\mathbf{D}^{-1} \mathbf{A}\right)^{l}}{2 m}-\frac{\mathbf{d d}^{T}}{4 m^{2}}\right) \mathbf{d}}{\mathbf{d}^{T}\left(\frac{\mathbf{D}}{2 m}-\frac{\mathbf{d d}^{T}}{4 m^{2}}\right) \mathbf{d}} .
$$

Given the connectedness of graph $G$, observe that the vector $\mathbf{q}$ is the unique stationary distribution. Being the matrix $\mathbf{P}$ is primitive, then $\lim _{l \rightarrow \infty} \mathbf{P}^{l}$ exists and:

$$
\lim _{l \rightarrow+\infty} \mathbf{P}^{l}=\left[\begin{array}{llll}
\mathbf{q} & \mathbf{q} & \ldots & \mathbf{q}
\end{array}\right]^{T}=\left[\begin{array}{llll}
\frac{\mathbf{d}}{2 m} & \frac{\mathbf{d}}{2 m} & \ldots & \frac{\mathbf{d}}{2 m}
\end{array}\right]^{T}=\frac{1}{2 m} \mathbf{1 d}^{T} ;
$$


we will call this matrix $\mathbf{P}^{\infty}$. Observing that

$$
\mathbf{D}_{\mathbf{q}} \mathbf{P}^{\infty}=\frac{\mathbf{d d}^{T}}{4 m^{2}}=\mathbf{q} \mathbf{q}^{T}
$$

equation 10 can be rewritten as:

$$
\rho_{w, l}=\frac{\mathbf{d}^{T}\left[\mathbf{D}_{\mathbf{q}}\left(\mathbf{P}^{l}-\mathbf{P}^{\infty}\right)\right] \mathbf{d}}{\mathbf{d}^{T}\left[\mathbf{D}_{\mathbf{q}}\left(\mathbf{I}-\mathbf{P}^{\infty}\right)\right] \mathbf{d}}
$$

then the assortativity of order $l$ vanishes as $l \rightarrow \infty$.

\section{Proof of Theorem 2}

Let $G=(V, E)$ be a simple connected graph. The coefficient $\rho_{c, \alpha}$ tends to $\rho$ as $\alpha$ tends infinity.

Proof. The matrix $\mathbf{H}_{\alpha}$ contains the reciprocal of $\alpha$-power of the distances between all couples of distinct nodes, adjacents or not. Being the graph $G$ connected, at least one path between every couple of nodes $i$ and $j,(i \neq j)$ exists, then $d(i, j) \geq 1$. In particular, $d(i, j)=1$ only if $i$ and $j$ are adjacents and in this case $\operatorname{dist}(i, j)^{-\alpha}=1$ for all $\alpha$, otherwise, $\lim _{\alpha \rightarrow+\infty} \operatorname{dist}(i, j)^{-\alpha}=0$. Then, when $\alpha$ approaches to infinity, $\mathbf{H}_{\alpha} \rightarrow \mathbf{A}, h=\sum_{i} \sum_{j} h_{i j} \rightarrow 2 m, \mathbf{q} \rightarrow \frac{\mathbf{d}}{2 m}$ and finally $\rho_{c, \alpha} \rightarrow \rho$.

\section{References}

[1] D. Alderson, J.C. Doyle, L. Li and W. Willinger, Towards a theory of scale free graphs. Definitions, properties and implications, Internet Mathematics, vol2, n.4, 431-523, 2005

[2] D. Alderson, J.C. Doyle, Lun Li and W. Willinger Supplemental material: the $\mathrm{S}(\mathrm{G})$ metric and assortativity, Internet Mathematics, vol2, n.4, 2005

[3] D.L. Alderson and L. Li Diversity of graphs with highly variable connectivity, Physical Review E 75046102 , 1-11, 2007

[4] Anklam P., KM and the social network, Knowl.Manag.Mag. 24-28, 2003

[5] F. Blochl, F. J. Theis, F. Vega-Redondo and E. O'N. Fisher, Vertex centralities in input-output networks reveal the structure of modern economies Physical Review E 83.046127, 2011 
[6] S. Borgatti, "Centrality and network flow" Social Networks 271: 55-71, 2005

[7] L. da F. Costa, F.A. Rodrigues, G.Travieso and P.R. Villas Boas, Characterization of complex networks: A survey of measurements, Advances in Physics 56 (1) 167-242, 2007

[8] E. Estrada, N. Hatano, and A. Gutierrez Clumpiness mixing in complex networks, J. Stat. Mech. pp. 1-25, P03008, 2008

[9] E.Estrada, Combinatorial study of degree assortativity in networks, Physical review E,84, 047101, 2011

[10] E. Estrada, The structure of complex networks, Oxford University Press, 2011

[11] F. Harary, Graph theory, Addison-Wesley, Reading, MA., 1696

[12] R.V. Hogg, J. McKean, and A.T. Craig Introduction to mathematical statistics, Pearson Education, 2005

[13] A. Mood, F.A. Graybill and D.C. Boes, Introduction to the theory of statistics, 1974

[14] R. Grassi, S.Stefani and A. Torriero, Extremal properties of graphs and eigencentrality in trees with a given degree sequence, Journal of Mathematical Sociology 34 (2) 115-135, 2010

[15] C.M. Grinstead and J.L. Snell, Introduction to probability, American Mathematical Soc, 1977

[16] I. Gutman, Degree-Based Topological Indices, Croat. Chem. Acta 86 (4) 351-361, 2013

[17] L. Lovász, Random Walks on Graphs: A Survey, Combinatorics, Paul Erdos is Eighty (Vol. 2) Keszthely (Hungary), pp. 1-46, 1993

[18] H. Li and M. Lu, The $m$-connectivity index of graphs, MATCH Commun. Math. Comput. Chem. 54, 417-423, 2005

[19] N. Litvak and R. Van Der Hofstad, Uncovering disassortativity in large scale-free networks, Physical Review E 87, 2013 
[20] N. Litvak and R. Van Der Hofstad, Dependency in random graphs with heavy-tailed degrees, AirXiv: 1202.3071v6, 2014

[21] M.M. Mayo, A. Abdelzaher and P. Ghosh, Long-range degree correlations in complex networks, Computational Social Networks 2:4, 2015

[22] M.E.J. Newman, S.H. Strogatz, and D. J. Watts, Random graphs with arbitrary degree distributions and their applications, Phys. Rev. E 64, 026118, 2001

[23] M.E.J. Newman, Assortative mixing in networks, Phys. Rev. Lett. $89.208701,2002$

[24] N. Daniel, Applied Linear Algebra, 1977

[25] R. Noldus. and P. Van Miegen, Assortativity in complex networks, JCN, doi: 10.1093/comnet/cnv005, 2015

[26] C. Orsini, M.M. Dankulov, A. Jamakovic, P. Mahadevan, P. Colomer-deSimón, A. Vahdat, K.E. Bassler, Z. Toroczkai, M. Boguñá, G. Caldarelli, S. Fortunato and D. Krioukov, How random are complex networks. arXiv preprint arXiv:1505.07503, 2015

[27] R Core Team, R: A language and environment for statistical computing. R Foundation for Statistical Computing, Vienna, Austria. URL https://www.R-project.org/, 2015

[28] J. Rada and O. Araujo, Higher order connectivity index of starlike trees, Discr. Appl. Math., 119, pp. 287-295, 2002

[29] M. Randić, Characterization of molecular branching, Journal of the American Chemical Society, 97 (23) pp. 6609-6615, 1975

[30] P. Van Mieghem, H. Wang, X. Ge, S. Tang, and F.A. Kuipers, Influence of assortativity and degree-preserving rewiring on the spectra of networks, The European Physcal Journal B, 2010

[31] I.G. Yero, J.A. Rodrguez-Velzquez, I. Gutman, Estimating the higher-order Randić index, Chem. Phys. Lett., 489, pp. 118-120, 2010 